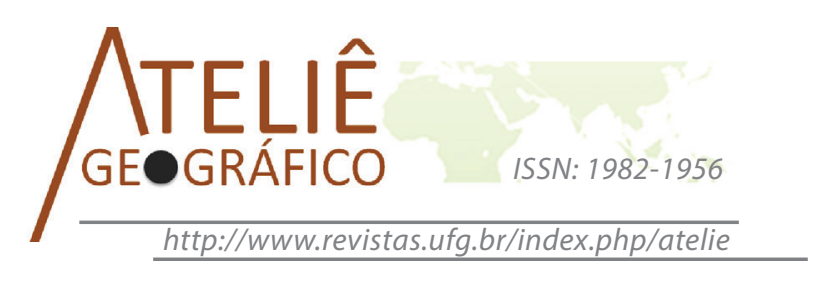

\title{
La construcción del conocimiento ambiental en México
}

\author{
A construção do conhecimento ambiental \\ no México
}

\section{The construction of environmental knowledge in Mexico}

\author{
Edgar Hernández-Quiroz, \\ Universidad Autónoma del Estado de México \\ edgarhquiroz@hotmail.com \\ Lilia Zizumbo-Villarreal \\ Universidad Autónoma del Estado de México \\ 1zv04@yahoo.com \\ Carlos Pérez-Ramírez \\ Universidad Autónoma del Estado de México \\ caperezr@uaemex.mx
}

\begin{abstract}
Resumen
Frente a la complejidad ambiental actual, en México han surgido numerosos programas de estudios de nivel superior y posgrado, así como diversos proyectos de investigación que pretenden su abordaje desde las ciencias ambientales, persiste un enfoque sumario y taxativo de diferentes disciplinas y campos de conocimiento con limitado aporte a la construcción de un nuevo proyecto transdisciplinario. Frente a este escenario, el trabajo tuvo como objetivo analizar el desarrollo del conocimiento ambiental en México, a partir de su conformación ontológica, epistemológica y metodológica. Para ello, se analizó la construcción del conocimiento ambiental desde la filosofía de la ciencia, considerando el conocimiento sobre el ser científico, la construcción del conocimiento y los procesos a partir de los cuales se ha construido. Se concluye que el enfoque especializado desde el cual se ha construido el conocimiento ambiental, restringe el desarrollo de nuevas aproximaciones, principios y perspectivas para la comprensión del mundo.

Palabras clave: conocimiento ambiental, ontológico, epistemológico, metodológico, teoría del conocimiento.
\end{abstract}




\begin{abstract}
Resumo
Diante da complexidade do ambiente atual, houve numerosos estudos de nível superior e pós-graduação no México, bem como vários projetos de pesquisa que visam a sua abordagem nas ciências ambientais. Apesar de oferecer uma abordagem prescritiva em diferentes disciplinas e áreas do conhecimento, ainda se observa a existencia de uma contribuição limitada para a construção de um novo projeto transdisciplinar nesse campo. Diante desse cenário, o estudo teve como objetivo analisar o desenvolvimento da consciência ambiental no México, a partir de sua conformação ontológicas, epistemológicas e metodológicas. Para fazer isso, a construção do conhecimento ambiental foi analisada a partir da filosofia da ciência, considerando o conhecimento científico sendo, a construção do conhecimento e os processos a partir do qual ele é construído. A análise revela que a abordagem especializada, a partir da qual se construiu o conhecimento ambiental, restringe o desenvolvimento de novas abordagens, princípios e perspectivas para a compreensão do mundo.
\end{abstract}

Palavras-chave: conhecimento ambiental, ontológico, epistemológico, metodológico; teoria do conhecimento.

\begin{abstract}
Given the current environmental complexity, there were many studies in Mexico at a tertiary and graduate level, as well as numerous research projects that have sought to approach this issue within the environmental sciences. Despite offering a prescriptive approach and knowledge with regards to different disciplines, it is still observed a limited contribution towards the construction of a new transdisciplinary project in this field. Regarding this scenario, the study aims to analyze the development of environmental awareness in Mexico from its ontological, epistemological and methodological conformation. To do this, the construction of environmental knowledge is analyzed from a philosophy of science perspective by considering the scientific knowledge as the result of the construction of knowledge and of processes whereby it was built. The analysis reveals that the specialized approach, through which the environmental knowledge was conceived, has restricted the development of new approaches, principles and perspectives for understanding the world.

Keywords: environmental, ontological, epistemological, and methodological knowledge; theory of knowledge.
\end{abstract}

\title{
Introducción
}

El conflicto ambiental imperante coexistente entre la sociedad mundial y sus vínculos con la naturaleza, se percibe a través de la pérdida de la biodiversidad, deforestación, desertificación, agotamiento de la capa de ozono, sobreexplotación de los recursos, destrucción del hábitat, introducción de especies exóticas, aislamiento, contaminación ambiental, explosión demográfica, consumismo, calentamiento global y cambio climático. Siendo además de relevancia los que ocurren a nivel local y regional como: contaminación atmosférica, contaminación de las aguas marinas o continentales, contaminación auditiva, contaminación visual, pérdida de suelos, generación de residuos sólidos municipales (RSM) y degradación de los ecosistemas estratégicos. 
Esta problemática ambiental latente a gran escala la cual enfrenta la sociedad actual casi en todos los niveles, obliga a la imperiosa necesidad de comprender y explicar la relación del hombre con el medio que lo rodea, así como los procesos inmersos en la misma que inciden en el deterioro e impacto ambiental con la finalidad de buscar posibles alternativas de solución para implementar medidas correctivas que favorezcan la conservación y el adecuado aprovechamiento de los recursos. De esta forma en México han surgido investigaciones y trabajos especializados orientados al análisis de la correlación sociedad - naturaleza.

Si bien dichos estudios se enfocaban inicialmente al estudio de las condiciones biofísicas, que desde el punto de vista aristotélico se orientaban exclusivamente al estudio del aire, suelo, fuego y recursos fósiles como fuente de energía; aunque también con amplia incidencia en los recursos hídricos, forestales, agropecuarios y los residuos. En la actualidad la perspectiva galileana de análisis se ha ampliado hacia los aspectos sociales pretendiendo asumir una concepción integral del ambiente que retome la dinámica de las interrelaciones de elementos naturales y elementos sociales, donde el ser humano y sus diferentes niveles de articulación económica, política, social y cultural constituyen un elemento indisoluble de esa red de interacciones.

De esta forma, se pretende superar el enfoque unitario o multidisciplinario en los que se involucran la participación de diversas disciplinas afines a las ciencias naturales o sociales para el abordaje del ambiente, hacia la construcción de un nuevo campo de estudio y acción que permita la integración interdisciplinaria e incluso transdisciplinaria, que aspire a la conformación de una nueva ciencia ambiental, como el eje central de los procesos y problemas asociados a la relación hombre naturaleza. Una visión holística donde la realidad no está constituida por cosas que tengan límites definidos, sino como una totalidad de campos de acción que se interfieren, donde todas las cosas rebasan sus propios límites estructurales y hay una tendencia a ir más allá de sí mismas (SOLÍS et al., 2003, p. 33).

Es obvio que para traspasar evidentemente la problemática ambiental existente y plantear alternativas de solución con respecto a esta, es necesario comprender los alcances del propio conocimiento ambiental, lo cual conlleva a reflexionar sobre la forma como se han entendido los objetos de conocimiento puestos en evidencia por los problemas ambientales en México, la forma en que éstos han sido abordados por las diferentes disciplinas y áreas del saber, el avance en la construcción de un área emergente del conocimiento denominada ambiental, así como el diálogo de saberes interdisciplinarios surgidos desde las llamadas ciencias ambientales, que si bien están formadas en las disciplinas tradicionales, las fortalecen y amplían frente a este nuevo objeto de conocimiento.

Por tal motivo, el objetivo de este trabajo se enfoca al análisis del desarrollo del conocimiento ambiental en México, a partir de su conformación ontológica, epistemológica y metodológica. Para ello, se analizó la construcción del conocimiento ambiental desde la filosofía de la ciencia, considerando el conocimiento sobre el ser científico, la construcción del conocimiento y los procesos a partir de los cuales se ha construido. 
El documento se estructura en cinco apartados, en el primero se esboza la concepción de la relación sociedad-naturaleza que incidió en el modo imperioso de comprender y explicar la relación del hombre con el medio que lo rodea dando por consecuencia el surgimiento de un campo disciplinar emergente conformado por las ciencias ambientales. En una segunda sección se presentan aproximaciones al conocimiento ambiental desde la teoría del conocimiento, abordándose la identificación de los procesos de construcción del conocimiento ambiental en México. La tercera sección está conformada por tres subsecciones en donde se caracterizan los procesos de construcción del conocimiento ambiental en México desde la filosofía de la ciencia, examinándose la propia construcción del conocimiento científico desde lo ontológico, lo epistemológico y lo metodológico. En el cuarto apartado se habla sobre la construcción del conocimiento ambiental en donde se caracteriza este a través de los procesos de apropiación, construcción y generación del mismo. Finalmente se presentan las conclusiones así como los aspectos para el acercamiento a la identificación de los procesos de construcción del conocimiento ambiental y lo que caracteriza a un conocimiento científico como ambiental.

\section{Aproximaciones al conocimiento ambiental desde la teoría del conocimiento.}

De acuerdo con la teoría del conocimiento, éste se entiende como el proceso que se reproduce en el pensamiento humano, condicionado por las leyes del acontecer social y ligado firmemente a la actividad práctica, que permite alcanzar la verdad objetiva. Desde el positivismo o visión analítica de la ciencia, como por el marxismo o teoría crítica de la ciencia, se reconoce la inherente presencia de dos elementos relacionados entre sí (sujeto - objeto), en el que la actividad de un sujeto permite aprehender de un objeto, pero desde la visión hermenéutica de la ciencia, se propone otro tipo de relación (sujeto - sujeto) (TAMAYO, 2011, p.13).

En el caso del conocimiento ambiental, se pueden presentar ambas relaciones, donde en el proceso del conocimiento, el sujeto que observa tiene las siguientes características: observa, aprende, capta, analiza. Las cuales determinan que su posición frente al objeto observado se encuentre por arriba de este. Por otro lado, el objeto que es motivo de revisión es: observado, aprendido, captado y analizado; se dice que está por debajo del observador. Además el objeto a observar también puede ser un sujeto y presentar las mismas características de un objeto observado, lo que Durkheim llamo la "cosificación" del sujeto. Sin embargo, también es posible valorar la hermenéutica que se contrapone a dicha corriente descriptiva, y que busca el conocimiento a través de la comprensión e interpretación de los sentidos en la relación sujeto - sujeto ambiental, sin la pretensión de resolverlas en ningún tipo de síntesis unificadora (CARVALHO, 2000).

Además, es posible analizar fenomenológicamente el proceso del conocimiento ambiental, el cual aspira a aprehender la esencia general en el fenómeno concreto. No trata de establecer lo que es propio de un conocimiento, ni de describir un proceso de conocimiento determinado, sino lo que es esencial a todo conocimiento, en qué consiste su estructura general donde sujeto y objeto están en una interrelación que se enriquece mutuamente. 
Para la identificación de los procesos de construcción del conocimiento ambiental en México desde la filosofía de la ciencia, se contempla la propia construcción del conocimiento científico desde lo ontológico o el conocimiento sobre el ser científico, lo epistemológico que aborda la construcción del conocimiento, así como lo metodológico que revisa los procesos a partir de los cuales se ha construido ese conocimiento científico. Estos tres procesos, están mediados por el tipo de relación establecida. La relación que, históricamente, es la más fuerte, es la relación sujeto - objeto. Por eso la mayor parte del conocimiento ambiental construido en México es analítico y luego crítico. La que está poco presente es la relación sujeto - sujeto, por lo que es poco el conocimiento ambiental producido en términos hermenéuticos. Se tiene entonces la necesidad de discutir lo anterior en este documento referenciándolo en las ciencias ambientales y en una ciencia ambiental. Por lo anterior es imperioso considerar lo siguiente: ¿qué caracteriza un conocimiento como ambiental? y ¿cuáles son los principales problemas teóricos y metodológicos de la construcción del mismo? Se puede decir entonces que los tres problemas fundamentales en la construcción del conocimiento ambiental son: primeramente el carácter de ese conocimiento, en segundo término ¿Cómo se le considera? y por último, la forma en cómo se construye.

\section{Lo ontológico}

Con relación a lo ontológico, se asume como el adjetivo que indica que algo es relativo o perteneciente a la ontología, es decir, a la rama de la filosofía de la ciencia que compone el conocimiento científico, y busca determinar las categorías fundamentales de la existencia y la realidad, así como la manera en que estas se relacionan entre sí. Para Husserl (1982) la ontología es la ciencia de las esencias y son de dos tipos: la formal que se encarga de estudiar a las esencias formales, es decir, a las propiedades de todas las esencias. Y en segundo término tenemos a la ontología material, la cual aborda las esencias materiales y se restringe según los modos de sus objetos. Por lo tanto, se les conoce también como ontologías regionales. Evidentemente la ontología formal abarca todas las materiales e incluso las del ser (HARTMANN, 1986).

La Ontología entonces, se ocupa del estudio del ente en cuanto tal, es decir, del ente en cuanto que es ente. No es materia de la ontología establecer que ente es este o aquel ente, sino de la determinación de lo que hace que éste y aquél sean considerados como entes. Si afirmamos que ente es cada cosa que es, entonces podemos preguntarnos qué es aquello en lo que todos los entes coinciden, qué es lo que tienen todos ellos en común, cuáles son sus notoriedades o características comunes. Ante esa interrogante, podemos contestar que los entes, considerados como entes, sólo tienen una característica en común: el ser; lo único que podemos decir de todos, independientemente de sus variaciones, es que son (HUSSERL, 1982 y 2005).

Es conveniente entonces tener una idea de cómo desde lo ontológico se piensa lo que es el conocimiento ambiental, ahí no hay un acuerdo todavía. Existen corrientes dominantes en relación al conocimiento ambiental, pero no hay una idea única de lo que es. Haciendo una revisión encontramos diferentes posturas del conocimiento ambiental desde las ciencias naturales, sociales o desde las ciencias en general, Leff (1994) discute 
proponiendo los elementos para una ciencia holística; Alimonda (2002) analiza los cambios ambientales desde una perspectiva social y política; Escobar (2000), Alimonda (2002) y Leff (1986) buscan explicar el cambio ambiental a partir de procesos políticos y disputas sociales, materiales y simbólicas; Giancarlo Delgado (2010), Martínez Alier y Jordi Roca (2000) también discuten otra postura desde la ecología económica en donde el conocimiento ambiental tiene sus características desde una frontera inexistente definida entre la economía ecológica y la economía ambiental. Entonces podemos decir que hay corrientes dominantes con relación al conocimiento ambiental actual. No hay una sola idea del mismo, igual que en todas las demás ciencias sociales podemos hablar de varias corrientes de pensamiento de lo ambiental. Sin embargo, podemos decir que la esencia formal del conocimiento ambiental producido hasta el momento en México es: El ambiente.

Ahora bien, ¿Qué se entiende por ambiente en ese conocimiento producido, es decir, en las ciencias naturales ambientales, en las ciencias sociales ambientales y en la ciencia ambiental? ¿Es posible hablar de una ciencia ambiental? Considerando las aportaciones de Leff $(1975,1986,1994)$ se puede hacer una reconstrucción y entonces: determinar lo que es la ciencia ambiental, identificar el tipo ideal de ciencia ambiental, caracterizar los constituyentes simples de los que se compone el conocimiento ambiental que se ha dado y el tipo de conocimiento ambiental construido en México hasta hoy.

Las diferentes formas de abordar la problemática ambiental así como de la conceptualización generalizada de ambiente por parte de las diversas disciplinas científicas, surgidas a partir de los años setenta del siglo veinte, permitió conformar una nueva área de conocimiento denominada ciencias ambientales. Emergidas principalmente como una especialización de las ciencias naturales, de las cuales tomaron tanto sus concepciones teóricas como metodológicas a fin de acercarse al nuevo objeto de estudio, instituyeron en sus inicios un espacio emergente de conocimiento ambiental surgido al interés y a las aportaciones de las ciencias tradicionales por comprender, delimitar y definir el concepto de ambiente a fin de contribuir a las propuestas de alternativas de solución de la problemática ambiental.

En este contexto surgieron múltiples conceptos de medio ambiente, como se le denominó en México durante bastante tiempo al objeto de estudio de las ciencias ambientales. Luego, varios autores demostraron lo redundante e innecesario de esta expresión puesto que utiliza dos términos: medio y ambiente, que significan la misma idea, tanto en su etimología como en el sentido que habitualmente se les atribuye: lo que cerca o rodea algo, es decir, el entorno. Por esta razón desde hace ya varios años, en nuestro país se ha extendido el uso de la palabra ambiente en lugar de medio ambiente. Sin embargo, el término de medio ambiente prevalece sobre todo a nivel institucional ejemplo de ello es el Instituto Nacional de Ecología y Cambio Climático, organismo dependiente de la Secretaria de Medio Ambiente y Recursos Naturales (SEMARNAT).

Cabe señalar que las definiciones de ambiente tienen su origen en distintas disciplinas científicas tradicionales, por lo que fueron, y siguen siendo, muy diversas. Las más comunes entre la sociedad científica y académica mexicana que trabaja temas ambientales desde la perspectiva de las ciencias sociales, encontramos a la sociología, la antropología y la historia, las cuales entiende ambiente como el campo de interacciones 
o relaciones entre sociedad y naturaleza o entre cultura y ecosistema. De igual modo como lo mencionan Escobar y Flores (2010) es muy frecuente el concepto propuesto desde la ecología que define al ambiente como las condiciones y factores externos, vivos y no vivos, substancias y energía, que influyen en un organismo u otro sistema específico durante su periodo de vida. Esos factores hacen referencia a características climáticas, edáficas, bióticas y de otros tipos e influyen directamente en la supervivencia, crecimiento, desarrollo y reproducción de los organismos. Comprende, por tanto, el componente biótico y abiótico de la biosfera. La noción de medio ambiente, conceptualizada desde la visión de la geografía ambiental, se ha utilizado comúnmente para señalar al ambiente como el conjunto de condiciones extrínsecas de orden biológico, físico y social que envuelven, influyen o contextualizan las actividades humanas y es usado como medio, elemento o insumo para satisfacer las necesidades humanas que, de hecho, son culturales e históricas. Por lo tanto, la problemática ambiental se ha colocado por fuera del componente social y se visualiza como un problema del ecosistema.

Este concepto de ambiente también se ha definido institucionalmente en varios ámbitos. En un inicio la entonces Subsecretaría del Mejoramiento del Ambiente dependiente de la Secretaría de Salubridad y Asistencia lo definió como el entorno natural de la población humana. Posteriormente la Secretaria de Desarrollo Urbano y Ecología precisó su objeto de estudio como el entorno físico y biótico, su relación con el medio ambiente y las dinámicas que tal relación conlleva. Además, es común el uso del concepto de ambiente que propone el Programa de las Naciones Unidas para el Medio Ambiente (PNUMA) (NACIONES UNIDAS, 1972) como el conjunto de factores naturales, sociales, económicos y culturales que rodean al hombre. De lo anterior se distingue que las anteriores definiciones institucionales presentan un elemento en común $y$ este es el concepto de ambiente como entorno, el cual es manejado como entorno natural o al referirse a los seres humanos como entorno biofísico.

Uno de los riesgos de esta noción institucional de ambiente, es que la distinción entre sociedad y naturaleza, entre cultura y ecosistema, entre los seres humanos y su entorno natural se pueda interpretar como la separación de estos elementos de la relación dada por este binomio. Esta lectura corresponde a la ruptura que entre sociedad y naturaleza son propias de la cosmovisión ligada con las ciencias naturales, las cuales identifican a los seres humanos como sujeto y a la naturaleza como objeto para poner ésta al servicio de aquellos, justificando así prácticas sociales depredadoras del medio natural. Esta interpretación es la que continúa predominando en la actualidad, en gran medida apoyada por los aportes de las disciplinas científicas tradicionales con los que se intenta entender el ambiente y dar solución a los problemas ambientales.

\section{Lo epistemológico}

La epistemología es una disciplina que estudia cómo se genera y se valida el conocimiento de las ciencias. Su función es analizar los preceptos que se emplean para justificar los datos científicos, considerando los factores sociales, psicológicos y hasta históricos que entran en juego. Este concepto fue usado inicialmente por James Frederick Ferrier en su obra titulada Institutos de Metafísica (LALANDE, 1992). 
La epistemología estudia los problemas relacionados con el conocimiento propiamente científico y “...es esencialmente el estudio crítico de los principios, hipótesis y resultados de las diversas ciencias, destinado a determinar el origen lógico y el valor y alcance de las mismas... es el estudio del problema... es la teoría del conocimiento científico" (LALANDE, 1992). Es la parte de la filosofía que trata de los fundamentos y los métodos del conocimiento científico (HONDERICH, 2005). En ese sentido, podemos establecer de manera más clara que la epistemología de lo que se encarga es de abordar la teoría del conocimiento científico a través de la respuesta a diversas preguntas de vital importancia como las siguientes: ¿Qué es el conocimiento?, ¿Cómo llevamos a cabo los seres humanos el razonamiento? y ¿Cómo se construye nuevo conocimiento?

Al referirnos al estudio crítico de principios, hipótesis y resultados de las diversas ciencias, para determinar el origen lógico, el valor y alcance de las mismas, tenemos que aquí se encuentran los elementos básicos de una discusión epistemológica. ¿En qué principios está basado el conocimiento ambiental producido en México, desde las ciencias naturales, desde las ciencias sociales, desde la ciencia ambiental en general? ¿Qué hipótesis atienden esas ciencias? ¿Qué resultados teóricos, construcciones teóricas se han alcanzado desde esas ciencias?

La epistemología se ocupa de problemas tales como las circunstancias históricas, psicológicas y sociológicas que llevan a la obtención del conocimiento científico y los criterios por los cuales se lo justifica o invalida. De aquí podemos entonces definir el objeto de estudio de la epistemología como: la producción y validación del conocimiento científico. Por consiguiente la epistemología analiza los criterios por los cuales se justifica el conocimiento científico, además de considerar las circunstancias históricas, psicológicas y sociológicas que llevan a su obtención. Con respecto al conocimiento científico ambiental producido en México tenemos que hacer las siguientes interrogantes ¿En qué circunstancias históricas, psicológicas y sociológicas se ha producido ese conocimiento, desde las ciencias naturales, las sociales y desde la ciencia ambiental en general? Además de considerar que los conocimientos ambientales producidos en México ¿Son aceptados por las comunidades científicas mexicanas y las de otros países? y ¿Qué grado de validez o invalidez tienen?

Si admitimos que la epistemología es la disciplina de los fundamentos y métodos del conocimiento científico. Entonces su objeto de estudio es: la producción y validación de ese conocimiento. Para ello, es necesario realizar el estudio de las circunstancias de validez y legitimación de los enunciados a través de los cuales se produce el conocimiento científico. Además, considerar los contextos científicos, históricos y sociológicos que llevan a la producción del conocimiento y los juicios por los cuales se le justifica o invalida.

Por consiguiente, la epistemología señala como requisitos indispensables para su reconocimiento como verídico, sus posibilidades y sus conexiones con los objetos del conocimiento, entre otros aspectos su Naturaleza, Justificación y Validez.

La reflexión epistemológica hacia el interior de las ciencias se hace necesaria y se ostenta como una acción nueva e interesante en la epistemología contemporánea. De hecho durante el desarrollo de las ciencias, se presentan ciertas crisis como consecuencia 
de la interpretación de los datos experimentales o por las mismas contribuciones deductivas; ello obliga a una crítica de los conceptos, métodos y principios, para determinar su valor epistemológico (KUHN, 2004; PIAGET, 1975). Es importante, entonces, que las ciencias ambientales se reflexionen a sí mismas en términos de aquellas teorías tomadas a partir de la ciencia en general y adoptadas como fundamento teórico de ciencias en desarrollo y que aspiren a convertirse en una teoría general del conocimiento ambiental; fortalezcan los métodos que se han seguido para acercarse al objeto de estudio; qué los nuevos conocimientos que están generando en relación de la aplicación de la teoría y la metodología permitan explicar y comprender la problemática ambiental, sus formas de organización, aplicaciones, desarrollos y repercusiones, a fin de conformarse, finalmente, en una Ciencia Ambiental.

Los epistemólogos ambientalistas dicen que el gran problema de la ciencia ambiental es el que se han generado ciencias particulares o especializadas cuando se debe crear una ciencia general, ya que la tendencia ha sido crear nuevo conocimiento desde la especialización. Desde la ilustración el objetivo era tener el dominio de la naturaleza.

El problema epistemológico es ¿Cómo construir un conocimiento holístico cuando estamos acostumbrados a crear conocimiento especializado? Durkheim dice que hay que hacer a un lado las prenociones, entendidas estas como las nociones de sentido común tanto de la población investigada como del investigador (MARDONES y URSUA, 1994). Todas las ciencias actuales trabajan en términos especializados y en la medida de lo posible se siguen especializando. Existe una enorme construcción de conocimiento especializado como: Química ambiental, Geografía ambiental, Administración ambiental o Turismo ambiental, entre otros, lo cual nos lleva a una primer pregunta iqué han hecho los químico, los geógrafos, los administradores en construcción de conocimiento ambiental? Si han hecho trabajos técnico-instrumentales, entonces se habla en términos de ciencia y/o racionalidad técnico instrumental.

De los trabajos que se están haciendo, actualmente, en las ciencias ambientales se tocan cuatro aspectos fundamentales a saber:

- La construcción del objeto de estudio. Desde que posturas se construye este objeto emergente de la cuestión ambiental, tanto de los aportes de las ciencias naturales ambientales como de las socioambientales y sobre todo el identificar si esta construido desde la ciencia ambiental

- La construcción de la teoría: ¿Existe fundamentación epistemológica de esos trabajos? es necesario revisar en qué teorías se basan esas tesis, trabajos e investigaciones, y si hay un fundamento teórico desde la ciencia ambiental y reconocer el enfoque socioambiental que se utilizó. Identificar las teorías que están sirviendo para hacer esos estudios ambientales y las teorías que no son de corte ambiental que se están usando y la forma en que se adaptan como teorías de ciencias naturales o sociales aplicadas al estudio de la problemática ambiental.

- La metodología que se ha seguido sobre el objeto de estudio. Esto es cuáles metodologías se están usando, si es que se está trabajando igual que en la disciplina en general como sí es que la química ambiental, la geografía 
ambiental, por ejemplo, están tomando metodologías empleadas en la química general o en la geografía ambiental a fin de determinar que sí y que no se toma y que se genera. Siendo este último una metodología propiamente de una ciencia ambiental en sí.

- Un nuevo conocimiento construido sobre ese objeto de estudio. Que nuevos conocimientos se están generando en relación de la aplicación de la teoría y la metodología ambiental. Como por ejemplo que nuevo conocimiento se está generando sobre el manejo de lodos o remoción de suelos que no se sabía.

Se evidencia una estrecha influencia y relación desde el contexto internacional que se movilizan hacia los saberes ambientales construidos en México, ya que como menciona Garza (1999) aunque existían investigaciones pioneras a este respecto, surgen en México los trabajos realizados por Peñafiel y Arizpe a fines del siglo antepasado, los cuales solo señalan la poca higiene que prevalecía en la Ciudad de México debido a la falta de agua, drenaje y alumbrado público y que, además, los estudios de medio ambiente fueron de poca importancia antes de 1960 sobresaliendo los trabajos de: Enrique Beltrán (1958) con El hombre y su ambiente, estudio sobre el valle de México y el de Gustavo Viniegra y Humberto Bravo (1958) con Polución atmosférica en la Ciudad de México, informe preliminar.

Las ciencias ambientales como campo emergente del conocimiento comenzaron a explayarse tanto a nivel mundial como a nivel nacional a finales de la década de los sesentas del siglo XX, pero es a partir de 1973 que la investigación ambiental institucional en México se incrementa en forma gradual influenciada por las conferencias de las Naciones Unidas sobre el medio ambiente humano celebradas en Suecia en el año de 1969 y el PNUMA surgido en Estocolmo en 1972; así como la creación en ese año por parte del presidente de la República, Luis Echeverría, de un organismo denominado Subsecretaría del Mejoramiento del Ambiente dependiente de la Secretaría de Salubridad y Asistencia y en 1982, al iniciarse el sexenio del Lic. Miguel de la Madrid, se creó la Secretaria de Desarrollo Urbano y Ecología. Paralelamente en 1974 se crea en la Universidad Autónoma Metropolitana, unidad Azcapotzalco, el Centro de Estudios del Medio Ambiente y a instancias del gobierno federal se crean los siguientes centros de investigación: el Centro de Ecodesarrollo en la Ciudad de México; el Instituto de Recursos Bióticos de Xalapa, Veracruz y el Centro de Investigaciones Ecológicas del Sureste, en San Cristóbal de las Casas, Chiapas. De esta época ya se manifiestan preocupaciones por los académicos sobre el deterioro ambiental causado por el comportamiento humano, así las principales investigaciones que se realizan abordan los problemas de la contaminación y la degradación del medio ambiente considerando el problema de los residuos, el del agua, el manejo de la energía, el del ruido y el de la radioactividad atmosférica sobresaliendo los trabajos de Vizcaíno (1975), Leff (1975), Restrepo (1976), Bravo (1973) y Escobar (1973).

El inicio de la institucionalización de la investigación ambiental se visualiza con el establecimiento de organismos públicos descentralizados y financiados por el gobierno de México. El tema ambiental fomenta estudios que abordan la problemática ambiental enfocada hacia investigaciones y trabajos especializados del deterioro ocasionado a 
éste y la búsqueda de posibles soluciones (IIYINA et al., 2002; MORENO et al., 2002; LUNA y RAMÍREZ, 2004; RIVAS et al., 2003). En los cuarenta y cinco años que han transcurrido desde la conferencia de Estocolmo, se ha venido realizando éste proceso: de estudio, adquisición de experiencia y profundización en la temática ambiental y en sus posibles soluciones. Se ha presentado un incremento en los conocimientos sobre el origen y la dinámica de la problemática ambiental.

Es durante la década de los noventas que se incrementa la investigación ambiental pero no se identifican trabajos, en esta etapa, que analicen la cuestión ambiental utilizando su propia concepción teórica. Por lo que ante la necesidad de entender la complejidad del objeto de estudio e intervención propio de las ciencias ambientales, se hace necesario un nuevo tipo de saber, un nuevo tipo de ciencia en que la ciencia ambiental debe desarrollar nuevos perfiles de investigación apoyados en enfoques tanto interdisciplinarios como transdisciplinarios que reconozcan, además, la valía y trascendencia de otros tipos de conocimiento con las cuales se mantiene un permanente diálogo de saberes. En su actual proceso de evolución, en esa búsqueda de perfeccionamiento, las ciencias ambientales se fortalecen de los constantes intercambios, no sólo entre las diversas disciplinas científicas sino, también, entre los distintos géneros de producción de conocimiento. En ello radica la fortaleza y especificidad de sus nuevos métodos de trabajo. Esta condición es dada por una persistente división entre naturaleza y sociedad propia de la cosmovisión de una modernidad globalizante, que fragmenta los componentes constitutivos del mundo como base al modelo de desarrollo imperante, sustentado en la visión de apropiación de la naturaleza, motivado por el pragmatismo de las ciencias naturales que convierten a ésta en una especie de mercancía, cuyo precio depende de su escasez.

En consecuencia, con esta conceptualización, las acciones dirigidas a la solución de la problemática ambiental se activan en el recinto de lo tecnológico o de la generación de la normatividad vigente a fin de llevar acciones para proteger a los recursos o evitar la destrucción de los mismos, o de incentivos para originar directivas propias hacia acciones remediales o de la normatividad vigente. Puesto que el ambiente se reduce al entorno biofísico que sirve de soporte a la actividad humana, los problemas ambientales se entienden como todo efecto que se manifieste en este entorno, como agotamiento de un recurso, daño o contaminación (BARKIN, 1989). De esta forma, los campos de conocimiento que se ocupan del ambiente corresponden a las ciencias naturales, básicas o aplicadas, y a las ingenierías; poco o nada intervienen en esto las ciencias sociales. Para tal marco ideológico, solo bastó con fomentar acciones para regular, prohibir o incentivar conductas y dar soluciones técnicas dirigidas a resolver dichos problemas.

De los trabajos que se están haciendo, actualmente, en las ciencias ambientales en México existe un conocimiento construido sobre ese objeto de estudio, además de nuevos conocimientos generados en relación de la aplicación de la teoría y la metodología ambiental. De aquí que de acuerdo con el estado del conocimiento ambiental en México desarrollado por Hernández (2008), se tienen identificados quince grupos que integran la panorámica ambiental sobre el conocimiento construido en México con sesenta y tres líneas de investigación que son:

- Cambio Climático (variación y cambio climático; captura de carbono; servicios ambientales; y gases y efecto invernadero). 
- Contaminación (en agua; atmosférica; y en suelo).

- Daños a la Salud (por contaminantes químicos; y por tabaquismo).

- Desarrollo Sustentable (interpretación, políticas, análisis e indicadores de desarrollo sustentable; metodología, taxonomía y modelos de desarrollo sustentable; desarrollo y manejo sustentable de recursos hidrológicos; agricultura sustentable; sustentabilidad agropecuaria; desarrollo forestal sustentable; y ecoturismo).

- Educación Ambiental (educación formal; educación no formal; y educación formal y no formal).

- Epistemología Ambiental (interpretación y análisis del desarrollo sustentable; conocimiento y análisis de la cuestión ambiental; población, emigración y ambiente; desarrollo de la sociología ambiental; estrategias para la conservación de ecosistemas y deterioro ambiental; metodología para estudios ambientales por daños a la salud; y economía ecológica y política ambiental.

- Gestión Ambiental (estrategias de gestión ambiental; planeación ambiental participativa; y gestión ambiental transfronteriza).

- Impacto Ambiental (Ecorrehabilitación, comercio y medio ambiente; deterioro ambiental y movimientos sociales; turismo e impacto ambiental; expansión urbana y actores sociales; niveles de desarrollo, bienestar social e influencia demográfica en el medio ambiente; conformación del índice de deterioro ambiental; $y$ industria petrolera).

- Legislación Ambiental (leyes y reglamentos; normas oficiales mexicanas; conservación de la naturaleza; y cuotas e impuestos en la protección del medio ambiente).

- Ordenamiento Ecológico (regionalización y ordenamiento ecológico; análisis geosistémico y regionalización geomorfológica; y enfoque morfoedafológico para evaluación de oferta ambiental).

- Política Ambiental (participación ciudadana en política ambiental; y política ambiental y dimensión regional).

- Reciclaje (costos y beneficios ambientales del reciclaje del aluminio; y la situación, producción y degradación de los envases de plástico (pet)).

- Residuos Peligrosos (manejo de residuos sólidos municipales; inventario y bases de datos de residuos peligrosos; residuos industriales peligrosos; biodegradación de residuos; y evaluación del riesgo para la salud por residuos).

- Riesgo Ambiental (análisis y evaluación del riesgo; cartografía morfogenética, geomorfología aplicada y remoción de masa; evaluación del riesgo por sustancias tóxicas; riesgo por inundaciones; y riesgo ambiental por organismos genéticamente modificados). 
- Tecnología Ambiental (química verde y tecnología aplicada; tratamiento de aguas y/o lodos residuales; modelos ambientales; biorremediación; sistemas energéticos; y adsorción y biosorción).

Fuente: Elaboración propia con base en investigación documental tomado de (HERNÁNDEZ, 2008).

Este conocimiento construido sobre la temática ambiental se centra mayoritariamente en el análisis de aspectos relacionados con: daños a la salud por contaminantes químicos y por tabaquismo; con la contaminación atmosférica, en agua y en suelo identificándose los principales contaminantes; en desarrollo sustentable desde la interpretación y análisis hasta propuestas hacia futuro; en tecnología ambiental considerando los diversos estudios para la solución de la problemática ambiental desde el punto de vista de la tecnología aplicada y en legislación ambiental indicando tanto la normatividad vigente como diversas propuestas para reglamentar y acotar el marco jurídico para el quehacer ambiental.

También hay un conjunto de temas como los análisis del cambio climático, la educación, gestión, impacto, riesgo y servicios ambientales así como la epistemología ambiental y el ordenamiento ecológico que son abordados en su mayoría desde la perspectiva de diversas disciplinas de tipo natural y muy escasamente por aquellas de tipo social. Esto último se puede atribuir a que no ha habido una actividad sostenida debido al reducido número de investigadores sociales, así como por la falta de formación de recursos humanos. Por otra parte, es evidente el gran involucramiento de las universidades y centros de investigación superior. Esto es, en cierta manera un hecho alentador, ya que cumplen con la expectativa ideal de tener una mayor participación en la investigación básica y aplicada, muy por encima de los organismos gubernamentales. Sin embargo, no se visualiza la misma participación en el caso del sector privado considerando la infraestructura de recursos materiales y humanos y la capacidad para interactuar de manera transdisciplinaria, tomando en cuenta los recursos económicos con que cuenta este sector, debiéndose quizá a que todavía no existen intereses comprometidos y una obligatoriedad en el orden ambiental por parte de las empresas.

Así pues la presencia de conocimiento ambiental en México durante las últimas décadas es el resultado de la confluencia de diferentes factores: de la entrada en vigor del protocolo de Kyoto, de una evolución del objeto de estudio de lo ecológico hacia lo ambiental, de un creciente interés hacia la temática ambiental por las instituciones educativas, gubernamentales y civiles y de una mayor colaboración entre estos tres niveles.

\section{Lo metodológico}

Otro aspecto relacionado a la epistemología es la metodología. De hecho para el metodólogo, el conocimiento no está subordinado a un juicio de valor, es considerado como información que está validada y aceptada por los científicos. La labor de la metodología es analizar en qué forma se puede expandir o acrecentar el conocimiento científico. La metodología como recurso específico derivado de una posición teórica 
y epistemológica, tiene su aplicación en la selección de técnicas específicas de investigación. Depende, además, de las premisas que el investigador considere válidas, ya que la actividad metodológica como herramienta eficiente del análisis de la realidad estudiada debe examinar en su totalidad a una problemática específica, además de ser sistemática, disciplinada y permitir un enfoque crítico.

La investigación puede presentar diversas metodologías, las cuales se agrupan en dos categorías: la metodología de investigación cuantitativa y la de investigación cualitativa. En la primera se accede a la información a través de la recolección de datos empíricos sobre las variables seleccionadas, y obteniendo conclusiones a través del comparativo estadístico; la segunda, se realiza a través de registros narrativos sobre los fenómenos investigados y obteniendo los datos por medio de entrevistas o técnicas nonuméricas, por lo que no se considera de importancia la cuantificación de datos y se analiza la intervariabilidad a partir de la observación además de considerar todos los contextos y escenarios presentes con respecto a un problema estudiado.

Ambas metodologías resultan valiosas y han ejecutado aportaciones al avance del conocimiento. Ninguna es evidentemente major que la otra, sólo constituyen diferentes aproximaciones al estudio de un fenómeno. Las dos metodologías en el estudio de la cuestión ambiental son complementarias; es decir, cada una sirve a una función específica para conocer un fenómeno, y para conducirnos a la solución de los diversos problemas y cuestionamientos. El investigador ambiental debe ser metodológicamente plural, y guiarse por el contexto, la situación, los recursos de que dispone, sus objetivos y el problema de estudio. Esto es, en efecto, una postura pragmática. En la investigación ambiental no se debe externar opinión sobre si una metodología es mejor que otra ya que hasta el momento las dos se han aproximado de distinta manera al objeto de estudio y cada investigador ha tomado diversas posturas ante la realidad y lo verdaderamente importante es que el conocimiento de lo ambiental es la meta de la ciencia ambiental. Existen en la actualidad varios trabajos de corte ambiental que utilizan ambas metodologías en algo que se ha dado en llamar el enfoque multimodal. (CHALLENGER, 2001; ALFIE y MÉNDEZ, 2000; LARES y LÓPEZ, 2004; TOLEDO et al., 2004)

La construcción de una racionalidad ambiental implica transformaciones de los conceptos y métodos de diversas ciencias y campos disciplinarios del saber, en los sistemas de valores y las creencias de diversos grupos sociales (LEFF, 1994). Estas mutaciones ideológicas y epistémicas conllevan el análisis de complejos procesos que articulan los intereses de diversas áreas del conocimiento ligadas con la identificación, caracterización y apropiación de los distintos saberes dentro de los cuales se desenvuelven las ciencias ambientales.

Las técnicas descontaminantes, los procesos de reciclaje de desechos y residuos, y la innovación de ecotécnicas presentes en el abordaje de la temática ambiental han generado un sistema tecnológico para la solución de la problemática inherente al conflicto ambiental, pero poco han contribuido a transformar los principios teóricos y metodológicos de las ciencias ambientales. Esto no sólo demanda nuevas metodologías para la integración de los saberes existentes y la colaboración de diferentes disciplinas para la explicación de esta realidad compleja, sino que estimula la producción de nuevos conceptos, e incluso la transformación y ruptura de ciertos paradigmas establecidos 
del conocimiento ambiental (HERNÁNDEZ, 2008, p. 27). Estos cambios epistémicos no sólo dependerán del cuestionamiento externo a las ciencias, sino de las estructuras mismas del conocimiento en cada campo del saber, que las hacen más maleables o rígidas para incorporar y fusionar un conocimiento ambiental.

Desde la revolución industrial se ha tenido la percepción de que los recursos naturales son infinitos y que el medio natural tenía suficiente capacidad de recibir los desechos sin afectarlo. En los últimos años, las cuestiones ambientales comenzaron a tener presencia en la opinión pública, lo que llevó a los gobiernos al reconocimiento del problema y de lo limitado de los recursos naturales disponibles para satisfacer las demandas de la sociedad. Derivado de esto, fue necesario implementar y gestionar la existencia de una normatividad ambiental, que si bien en un principio se presentó con más optimismo que efectividad, impulsó la creciente revisión de la temática ambiental en materia legislativa. Dando por resultado algunas de las leyes con temática ambientalista como: Ley General del Equilibrio Ecológico y la Protección al Ambiente (LGEEPA), la cual esta orientada a la preservación y restauración del equilibrio ecológico, así como a la protección del ambiente; Ley General del Desarrollo Forestal Sustentable, tiene por objeto regular y fomentar la conservación, protección y restauración, entre otros, de los ecosistemas forestales del país; Ley General para la Prevención y Gestión Integral de los Residuos, tiene por objeto fomentar la gestión integral de los residuos peligrosos y de los residuos sólidos urbanos, y encargarse de su manejo; así como prevenir la contaminación de los sitios cercanos a dichos residuos; Ley General de la Vida Silvestre, Relativa a la conservación y aprovechamiento sustentable de la vida silvestre y su hábitat. Cabe mencionar también el establecimiento de normas, atributos, directrices o prescripciones aplicables a un producto, método de producción u operación a través de las Normas Oficiales Mexicanas (NOM) elaboradas por el Comité Consultivo Nacional de Normalización de Regulación y Fomento Sanitario con la intención de vigilar, regular y sancionar los procesos de producción de compuestos presumiblemente contaminantes. Condicionado por lo anteriormente expuesto surgieron las primeras investigaciones encaminadas al desarrollo y modernización de procesos y sustancias alternativas con menor incidencia de toxicidad con la intención de mitigar la emisión de contaminantes y dar cumplimiento al marco normativo de reciente creación.

Un ejemplo de lo anterior es la tecnología ambiental o Química Verde la cual consiste en el desarrollo de metodologías para modificar la naturaleza de los productos o procesos para reducir los riesgos que estos representan para la salud y el ambiente (YARTO et al., 2004). En México se han implementado tecnologías limpias con el fin de desarrollar procesos amigables con el ambiente para la fabricación de nuevas sustancias y productos; sin embargo, se observa la falta de coordinación y apoyo entre los diversos sectores, instituciones y actores involucrados.

Se aprecian al respecto varias líneas de investigación de las cuales sobresalen las siguientes: Química Verde y Tecnología Aplicada; Tratamiento de Aguas y/o Lodos Residuales; Modelos Ambientales; Biorremediación; Sistemas Energéticos; y la de Adsorción y Biosorción.

La proporción de trabajos ambientales que no utilizan datos o que lograron una primera aproximación al fenómeno estudiado se aprecia muy claramente inferior 
a la encontrada en aquellas que aplicaron únicamente análisis descriptivos o que correlacionaron variables. Uno de los rasgos distintivos de la publicación ambiental mexicana consiste en el predominio de los estudios transversales por sobre los de corte longitudinal. De hecho los tipos de análisis estadísticos empleados son un reflejo de los diseños aplicados; y en consecuencia se ha limitado la producción de conocimiento ambiental, lo que ha propiciado la integración de conocimiento desde las propias disciplinas tradicionales. Y aunque se evidencia un esfuerzo, éste es poco significativo, por lo tanto la teoría es pobre y las metodologías también. Se repiten modelos y técnicas. Además, debido a que la cuestión ambiental se continúa visualizando como efectos en lo biofísico, la metodología es principalmente tomada de las ciencias naturales o exactas y se percibe poca participación sobre lo social o lo realmente ambiental.

Finalmente, en estos trabajos predomina la utilización en mayor grado de técnicas de gabinete en donde se utilizaron datos o información generada por otras investigaciones que aquellas en donde los investigadores generaron sus propios datos a través de estudios de campo, encuesta e incluso de tipo experimental, hecho que sin duda está muy relacionado con las técnicas utilizadas.

\section{La construcción del conocimiento ambiental.}

El ambiente es el resultado de la interacción entre sociedad y naturaleza e implica una forma particular del ser social y del ser paisaje. Así, el objeto de estudio de las ciencias ambientales es en concreto el resultado de la interacción de estos dos entes: sociedad-naturaleza, que se manifiesta tanto en la transformación del ambiente como en la construcción de la institucionalidad social, científica, tecnológica y económica. En este sentido todas las ciencias y disciplinas son potencialmente ambientales, pues sin perder su objeto tradicional, se pueden enfocar a este nuevo campo de estudio, como la ingeniería ambiental, administración ambiental, economía ambiental, filosofía ambiental, economía ecológica, química ambiental, geografía ambiental. Pero todas ellas están orientadas hacia un conocimiento particular como resultado de una forma de caracterizar los conocimientos de forma más especializado, esto viene desde la Ilustración o el Iluminismo desde que se generaron las ciencias Humanísticas hasta nuestros días.

Resulta claro que los objetos de estudio e intervención de los que se ocupan las ciencias ambientales, emanadas de la especialización tanto de las ciencias naturales como de las sociales que han trasladado su objeto de estudio hacia lo ambiental, son de naturaleza compleja, puesto que abarcan dinámicas interrelacionales de las estructuras, procesos y funciones de ecosistemas y culturas. Pero la comprensión de esta complejidad y la posibilidad de intervenir eficazmente sobre ella son imposibles desde las disciplinas científicas tradicionales que mantienen un enfoque reduccionista y simplificador propio del modelo de las ciencias decimonónicas.

De algún modo, la complejidad de los problemas ambientales y del propio concepto de ambiente ha hecho más que evidente la incapacidad de las ciencias en general para cumplir con estos objetivos. La propia fragmentación y especialización del desarrollo de las ciencias naturales y sociales, deja a un lado diversos elementos y determinantes de la crisis ambiental actual, pues su enfoque positivista refiere a los objetos 
de estudio en términos físicos, empíricos y medibles, sin considerar las interrelaciones socioculturales desde otros enfoques comprensivos o críticos.

La investigación respecto a la orientación y producción del conocimiento ambiental da cuenta que la contribución a este es limitada. Por ello, es preciso contribuir en la generación de un nuevo tipo de pensamiento que traspase las limitaciones dadas por las disciplinas científicas tradicionales, naturales y sociales, llamadas actualmente ciencias ambientales, para ofrecer soluciones eficaces a la grave crisis ambiental que vivimos desde las últimas décadas del siglo pasado y para construir un concepto de ambiente que precise adecuadamente el objeto de estudio. Estos son los retos que deben ser asumidos por la ciencia ambiental.

- Construcción de objetos de estudio ambientales

- Construcción de teorías o de conocimiento ambiental

- Nuevas metodologías

- Nuevo conocimiento.

Esta es la etapa en que se encuentra actualmente el proceso histórico de consolidación de la nueva área de conocimiento que constituyen las ciencias ambientales. Una etapa consecuente inmediata será el abordar el objeto de estudio ambiental ya no desde la interdisciplinariedad de las ciencias ambientales sino desde la transdisciplinariedad de una nueva ciencia ambiental, con un objeto de estudio construido bajo una ontología y con una nueva epistemología ambiental.

\section{Conclusiones}

Para comprender la forma de construcción del conocimiento ambiental, es preciso reconocer su concreción como campo de estudio emergente para las ciencias naturales y sociales que lo abordan desde sus propios conceptos, pero también el actual traslado de sus métodos, teorías y conceptos para explicar la problemática ambiental, que dio paso al surgimiento de disciplinas como la ingeniería ambiental, administración ambiental, geografía ambiental, química ambiental, entre otras. Con la integración y consolidación de estas ciencias ambientales desde una perspectiva transdisciplinaria, se debería dar paso a la construcción de una ciencia ambiental, con sus teorías, métodos, conceptos y epistemología propios.

Con la finalidad de conocer la forma de construcción del conocimiento ambiental en México, este artículo retomó su concreción desde la filosofía de la ciencia a partir del estudio sobre el ser científico, la construcción del conocimiento y los procesos a partir de los cuales se ha construido.

A partir de la revisión de los trabajos que se están elaborando actualmente desde las ciencias ambientales en nuestro país, se identifica que corresponden en un $73 \%$ a las ciencias naturales, cuyo avance se cimenta en el trasladado de sus fundamentos teóricos y metodológicos para la interpretación de la complejidad ambiental. Por lo tanto existe una importante construcción de conocimiento especializado desde la química ambiental, 
geografía ambiental, administración ambiental o turismo ambiental, entre otros, enfocándose, principalmente, a trabajos técnico-instrumentales, lo que permite enunciar que en este conocimiento ambiental construido se habla más en términos de ciencia y racionalidad técnico instrumental.

La construcción epistemológica hacia el interior de las ciencias ambientales, se hace necesaria, pues si bien se han realizado importantes contribuciones desde la construcción de datos, procesos experimentales, aportaciones interpretativas, deductivas o perspectivas críticas, aún no se ha construído teorías propias de la ciencia ambiental. Las ciencias ambientales se han construido desde la ciencia en general y adoptadas como fundamento teórico de las ciencias tradicionales en desarrollo que aspiran a convertirse en una teoría general del conocimiento ambiental que permita explicar y comprender: la problemática actual, sus formas de organización, aplicación y desarrollo.

Por lo tanto, el conocimiento ambiental en México se ha venido construyendo desde la ciencia en general, que si bien se aborda desde un enfoque especializado, restringe el desarrollo de nuevas aproximaciones, principios y perspectivas para la comprensión del mundo. Frente a esta realidad, se hace necesario insistir en revisiones más profundas que permitan identificar tres aspectos fundamentales a saber: la construcción de la teoría, la metodología que se ha seguido sobre el objeto de estudio y el nuevo conocimiento construido sobre ese objeto de estudio. Por lo tanto, no hay nuevas aportaciones metodológicas para este nuevo campo de estudio.

Resulta ineludible avanzar en la construcción de un nuevo tipo de pensamiento que traspase las limitaciones dadas por las disciplinas científicas tradicionales, naturales y sociales asumidas como ciencias ambientales, para comprender la realidad y ofrecer soluciones a la complejidad ambiental que desde las últimas décadas del siglo pasado, ha deteriorado las condiciones biofísicas del planeta y la forma de vida para millones de personas. Estos retos deben ser asumidos en la etapa en que se encuentra actualmente el proceso histórico de consolidación de la nueva área de conocimiento que constituyen las ciencias ambientales. Una etapa que además deberá avanzar en la definición del objeto de estudio ambiental, ya no desde la interdisciplinariedad de las ciencias ambientales sino desde la transdisciplinariedad de una nueva ciencia ambiental, con un objeto de estudio soportado y construido en una nueva epistemología que responda a los saberes referidos ontológicamente y apoyados en marcos metodológicos propio de la ciencia ambiental.

\section{Referencias}

ALFIE, M.; MÉNDEZ, L. Industria maquiladora y deterioro ambiental: ¿problema social?. El Cotidiano, México, vol. 17, núm. 104, p. 60-75, 2000.

ALIMONDA, H. (coordinador). Ecología política: naturaleza, sociedad y utopía. Buenos Aires: CLACSO-CICCUS, 2002.

BARKIN, D. La degradación del medio ambiente y la transformación productiva en México; las contradicciones del manejo de la crisis. Relaciones: estudios de historia y sociedad, México, vol. 10, núm. 37, p. 5-23, 1989. 
BELTRÁN, E. El hombre y su ambiente, estudio sobre el Valle de México. México: Fondo de Cultura Económica, 1958.

BRAVO, H. Estudio comparativo y evaluación de la calidad del aire en la Ciudad de México. México: Secretaría de Salubridad y Asistencia, 1973.

CARVALHO, I. Los sentidos de lo ambiental: la contribución de la hermenéutica a la pedagogía de la complejidad. En: LEFF. E. (coord.) La complejidad ambiental. México: Siglo XXI Editores, 2000. Cap. III, p. 85- 105.

CHALLENGER, A. Estrategias para la conservación de Ecosistemas. Gaceta Ecológica, México, núm. 61, p. 22-29, 2001.

DELGADO, G.; GAY, C.; IMAZ, M.; MARTÍNEZ, A. (coords). México ante el cambio climático. México: CEIICH-PINCC-CCA-PUMA-UNAM, 2010.

ESCOBAR, A.; FLORES, A. Ecología y medio ambiente. México: M^ Graw Hill, 2010.

ESCOBAR, A. El lugar de la naturaleza y la naturaleza del lugar: ¿globalización o postdesarrollo?. En: LANDER, E. (comp.) La colonialidad del saber: eurocentrismo y ciencias sociales. Perspectivas Latinoamericanas. Buenos Aires, Argentina: CLACSO, 2000.

ESCOBAR, R. Las operaciones industriales como fuente de contaminación atmosférica. México: Secretaria de Salubridad y Asistencia, 1973.

GARZA, G. Cincuenta años de investigación urbana y regional en México, 1940 1991. México: El Colegio de México, 1999.

HARTMANN, N. Ontología. México: Fondo de Cultura Económica, 1986.

HERNÁNDEZ, E. EI estado del conocimiento en la investigación ambiental en México. Tesis de maestría en ciencias ambientales. UAEM, Toluca Estado de México, septiembre, 2008.

HONDERICH, T. (Editor). Enciclopedia Oxford de Filosofía. Madrid: Tecnos, 2005. HUSSERL, E. La idea de la fenomenología. Cinco lecciones. México: FCE, 1982.

HUSSERL, E. Ideas relativas a una fenomenología pura y una filosofía fenomenológica. México: I.I.F.-UNAM, 2005.

IIYINA, A.; VILLARREAL, J. A.; RODRÍGUEZ, J. Aprovechamiento del residuo de la industria papelera como vehículo de microorganismos en tareas de Biorremediación. Revista Internacional de Contaminación Ambiental. Distrito Federal México, vol. 18, número 2, p.81-89, 2002.

KÜHN, T. S. La estructura de las revoluciones científicas. México: FCE, 2004.

LALANDE, J. Diccionario filosófico. Madrid: Herder, 1992. 
LARES, O; LÓPEZ, A. Metodología de diagnóstico para el desarrollo sustentable. Revista del Centro de Investigación, Distrito Federal México, vol. 6, núm. 22, p. 27 $38,2004$.

LEFF, E. Hacia un proyecto de ecodesarrollo. Comercio exterior, Distrito Federal México, vol. 25, núm. 1, p. 84-92, 1975.

LEFF, E. (Coordinador). Los problemas del conocimiento y la perspectiva ambiental del desarrollo. México: Siglo XXI, 1986.

LEFF, E. (Coordinador). Ciencias sociales y formación ambiental. México: Gedisa, 1994.

LUNA, V.; RAMÍREZ, H. Medios de soporte alternativos para la remoción de fósforo en humedales artificiales. Revista Internacional de Contaminación Ambiental, Distrito Federal México, vol. 20, núm. 001, p. 31-38, 2004.

MARDONES, J. M.; URSUA, N. Filosofía de las ciencias humanas y sociales: materiales para una fundamentación científica. Coyoacán México D. F.: Fontamara, 1994.

MARTÍNEZ, J.; ROCA, J. Economía ecológica y política ambiental. México: PNUMA/FCE, 2000.

MORENO, J.; COLIN, A.; BALCÁZAR, M.; TAVERA, L. Feasibility analysis of a sewage sludge treatment by an irradiation plant in Mexico. Revista Internacional de Contaminación Ambiental, Distrito Federal México, vol. 18, núm. 004, p. 191-197, 2002.

NACIONES UNIDAS. Informe de la Conferencia de las Naciones Unidas sobre el Medio Humano. Estocolmo: ONU, 1972.

PIAGET, J. Naturaleza y método de la epistemología. Buenos Aires: Paidós, 1975.

RESTREPO, I. El ecodesarrollo y algunos problemas del sector agropecuario. Revista de Comercio Exterior, México, vol. 26, núm. 1, p. 9-16, 1976

RIVAS, L.; NEVÁREZ, V.; BAUTISTA, R. Tratamiento de aguas residuales de uso agrícola en un biorreactor de lecho fijo. Agrociencia, Texcoco México, vol. 37, núm. 002, p. 157-166, 2003.

SOLÍS, L. M.; GONZÁLEZ, M. A.; VILLAFAÑA, L. F. La Educación Ambiental. En: SOLIS, L. M.; LÓPEZ, J. (comp.) Principios Básicos de Contaminación Ambiental. Toluca Estado de México: UAEM, 2003. Cap. 2, p.15-36.

TAMAYO, M. El proceso de la investigación científica. México: Limusa, 2011.

TOLEDO, V.; ALARCÓN, P.; BARÓN, L. Revisualizar lo rural: un enfoque socioecológico. Gaceta Ecológica, Distrito Federal México, núm. 062, p. 7-20, 2002.

VINIEGRA, G.; BRAVO, H. Polución atmosférica en la Ciudad de México, informe preliminar. En: REUNIÓN ANUAL DE LA DIRECCIÓN DE HIGIENE 
INDUSTRIAL, Puebla, 1958. Memoria, México: Secretaría de Salubridad y Asistencia, 1958. p. 10-28.

VIZCAÍNO, F. La contaminación en México. México: FCE, 1975.

YARTO, M.; GAVILÁN, A.; MARTÍNEZ, M. A. La química verde en México. Gaceta Ecológica, Distrito Federal México, núm. 72, p. 35-44, 2004.

EdgAR HERnÁNDEZ-Quiroz,

Doctorante en Ciencias Ambientales - Universidad Autónoma del Estado de México.

Paseo Colon esq. Paseo Tollocan s/n. C.P. 50120, Toluca, México.

E-mail: edgarhquiroz@hotmail.com

LiLia ZizumBo-VillarReAL

Doctora en Sociología por la UNAM. Profesora Investigadora de Tiempo Completo de la Facultad de Turismo y Gastronomía en La Universidad Autónoma del Estado de México. Campus CU Cerro de Coatepec s/n, Ciudad Universitaria. CP 50100.Toluca, México.

E-mail:1zv04@yahoo.com

CARlos PÉrez-Ramírez

Estudiante del Doctorado en Ciencias Ambientales de la Universidad Autónoma del Estado de México. Facultad de Planeación Urbana y Regional.

Mariano Matamoros esq. Paseo Tollocan s/n, C.P. 50120, Toluca, México.

E-mail: caperezr@uaemex.mx

Recebido para publicação em julho de 2015 Aprovado para publicação em novembro de 2015 